\title{
Response of the G2-prophase checkpoint to genotoxic drugs in lymphocytes from healthy individuals
}

\author{
Juana Pincheiraa, Consuelo de la Torre ${ }^{\mathrm{b}}$, Natalie Rodríguez ${ }^{\mathrm{c}}$, Carlos Y Valenzuela ${ }^{\mathrm{a}}$ \\ a Human Genetics Program, ICBM, School of Medicine, University of Chile \\ b Centro de Investigaciones Biológicas, CSIC. Ramiro de Maeztu, 9. Madrid-28040, Spain \\ c Unit of Hematology-Oncology, Department of Pediatrics, Dr. Roberto del Río Hospital. Prof. Alberto Zañartu 1085 - Santiago, Chile.
}

\begin{abstract}
We analyzed the in vitro effects of the anti-tumoral drugs doxorubicin, cytosine arabinoside and hydroxyurea on the G2-prophase checkpoint in lymphocytes from healthy individuals. At biologically equivalent concentrations, the induced DNA damage activated the corresponding checkpoint. Thus: i) there was a concentration-dependent delay of G2 time and an increase of both the total DNA lesions produced and repaired before metaphase and; ii) G2-checkpoint adaptation took place as chromosome aberrations (CAs) started to appear in the metaphase, indicating the presence of unrepaired double-strand breaks (DSBs) in the previous G2. The checkpoint ATM/ATR kinases are involved in DSB repair, since the recorded frequency of CAs increased when both kinases were caffeine-abrogated. In genotoxic-treated cells about three-fold higher repair activity was observed in relation to the endogenous background level of DNA lesions. The maximum rate of DNA repaired was 3.4 CAs/100 metaphases/hour, this rise being accompanied by a modest 1.3 fold lengthening of late G2 prophase timing. Because of mitotic chromosome condensation, no DSBs repair can take place until the G1 phase of the next cell cycle, when it occurs by DNA non-homologous end joining (NHEJ). Chromosomal rearrangements formed as a consequence of these error-prone DSB repairs ensure the development of genome instability through the DNA-fusion-bridge cycle. Hence, adaptation of the G2 checkpoint supports the appearance of secondary neoplasia in patients pretreated with genotoxic drugs.
\end{abstract}

Key words: DNA damage and repair; G2-prophase checkpoint adaptation; premitotic DSBs and chromosomal aberrations; anti-tumoral genotoxic drugs

\section{INTRODUCTION}

In proliferating cells, checkpoints are protein-to-protein pathways that sense a risky condition for a cell facing its irreversible transition to the following cycle phase. They then transduce antimitogenic signals to transiently delay such phase transition until it becomes safe for the cell. Most checkpoints are activated by DNA structural changes, such as those produced by deficiencies in DNA decatenation, replication or repair, although they also respond to other conditions, such as faulty achievement of a certain cell mass threshold for mitosis to be initiated. The checkpoint pathways activated by DNA damage are formed by early (proximal) kinases, which senses and initiates signaling, intermediate transducers of antimitogenic signals, and downstream effector kinases, such as CHK1, CHK2 and MAPKAPK2 (Zhang et al., 2008)

Checkpoints, when activated by DNA lesions, initiate two sub-pathways. The first stimulates the transcription of genes coding for enzymes responsible for DNA repair (Zhou and Elledge, 2000). The second subpathway blocks CDK machinery, usually by inhibiting Cdc25 phosphatase and then preventing the mitogenic dephosphorylation of the T14/ Y15 residues in the CDK catalytic subunit (Sanchez et al., 1997). As a consequence of the latter checkpoint subpathway, the proliferating cell has more time to complete the missing requirement that activated the checkpoint, increasing the total amount of DNA repair that takes place before the irreversible transition to the following cycle phase.
Finally, when DNA damage overrides the repair ability of a particular cell, the checkpoint pathway acquires a third function. It then induces apoptosis by activating the corresponding program (Bernstein et al., 2002). This latter function is the only one that supports a tumor suppressor function of checkpoint activation.

Though the checkpoint stringency differs in the different cycle phase transitions they control, any checkpoint-induced block is always transient. Thus, such a block is spontaneously overridden after some time, even though the requirement that activated the checkpoint remains partially unfulfilled (Paulovich et al., 1997). This property is known as checkpoint adaptation.

Adaptation to the G2 checkpoint is unique as the DNA lesions that are not repaired in G2-prophase will be processed to chromosomal aberrations (CAs) (Lee et al., 1997). Thus, both premitotic chromatid individualization and mitotic chromosome condensation result in the DNA lesions present in late G2 remaining unrepaired until the G1 of the subsequent cell cycle. In G1, the absence of the sister chromatid with an undamaged copy of lost or damaged DNA sequences results in DSB repair taking place by a non-homologous recombination mechanism. As a consequence, chromosomal rearrangements are induced, which initiate the DNA breakage-fusion-bridge cycle that supports the development of genomic instability to be clonally inherited by the progeny of each cell adapted to the G2 -prophase checkpoint (McClintock, 1984).

In the present report, we study the response of lymphocytes from healthy individuals in vitro exposed 
to four moderate doses of three different genotoxic drug treatments performed specifically during the G2-prophase. The treatments comprise the anthracycline antibiotic drug doxorubicin/adriamycin (DOX), the antimetabolite cytosine arabinoside, also known as arabinosyl-cytosine and cytarabine (ara C), which incorporates into DNA, and finally hydroxyurea (HU), which prevents the formation of deoxyribonucleotides by inhibiting the ribonucleotide reductase enzyme. These three antitumoral drugs behave as efficient genotoxic and clastogenic agents that modify DNA structure and function in the cells that are replicating their DNA. Nevertheless, each of these drugs displays other drug-specific effects. Hence, DOX generates reactive oxygen species (Gajewski et al., 2007), and prevents the binding of topoisomerase II to DNA because of its intercalation among adjacent DNA bases. On the other hand, ara $\mathrm{C}$ when incorporated into DNA, prevents elongation of the freshly initiated DNA chains, and also inhibits excision repair by blocking the action of $\alpha, \delta$ and $\varepsilon$ DNA polymerases (Mirzayans et al., 1993), whereas HU inhibits both nuclear DNA replication and repair (Müller et al., 2005).

Our results showed that in proliferating lymphocytes from healthy donors, the three antitumoral drugs assayed are also genotoxic when applied to late G2-prophase when cells perform their post-replication DNA repair. The frequency of chromosomal aberrations recorded in the metaphase increased with treatment concentration in the previous G2-prophase. The induced block that the checkpoint induces in G2-prophase progression was consistently overridden before DNA repair was complete (checkpoint adaptation). These data altogether support that genotoxic drugs, because of their effect on postreplication DNA repair, activate the G2-prophase checkpoint, which in turn promotes the development of genomic instability instead of preventing it.

\section{MATERIALS AND METHODS}

The study included 30 control individuals in good health, from 20 to 40 years of age ( 15 males and 15 females). These controls were assigned into three groups of ten individuals; each group was used to evaluate the in vitro effects of DOX, araC or HU separately. To each drug assay, the blood samples $(10 \mathrm{~mL})$ from each of the ten individuals was used to set up 25 whole blood lymphocyte cultures. Ten of them were used to evaluate chromosomal aberrations and the remaining 15 to estimate G2 timing.

For each culture, $0.4 \mathrm{~mL}$ of blood was added to $5 \mathrm{~mL}$ of TC medium RPMI 1640 (Gibco), supplemented with 20\% heat-inactivated fetal bovine serum (Gibco), $1 \%$ penicillin and streptomycin and 2\% phytohemagglutinin (M Form, Gibco). Cultures were incubated at $37^{\circ} \mathrm{C}$ for $72 \mathrm{~h}$. Then, the cells were blocked in metaphase by adding $0.2 \mu \mathrm{g} / \mathrm{mL}$ colcemid (Karyo Max, Gibco) during the last $3 \mathrm{~h}$ before harvesting.

\section{Drug solutions and treatments}

The three antitumoral drugs assayed (DOX, ara C and HU) were all from Sigma. Their CAS registry numbers were 69-749, 127-07-1 and D1515, respectively. These drugs, as well as caffeine (Merck, CAS 58-08-2), the inhibitor of the checkpoint ATM and ATR kinases (Sarkaria et al., 1999), were diluted in TC medium RPMI 1640 to obtain the corresponding stock solutions. They were diluted again to get the concentrations specified for each treatment in the Results section.

\section{Evaluation of chromosomal aberrations}

Two of the 10 cultures set up were used to determine the frequency of CAs under control conditions. These CAs represent the basal metabolic DNA damage without any genotoxic treatment. They are displayed in the first row of Table 1, either when the ATM and ATR kinases activities were suppressed by caffeine (column B in Table 1) or when they remained functional, i.e. in the absence of added caffeine (column A in the same Table).

The other 8 cultures were divided into 4 pairs and each treated with one of the four drug concentrations assayed. Again, one of each pair of cultures was used to determine the yield of chromosome aberrations induced by the drugs (column A), while the other was additionally treated with $5 \mathrm{mM}$ caffeine in order to inhibit the activity of the checkpoint kinases ATM/ATR during G2-prophase, i.e. in the last $3 \mathrm{~h}$ before metaphase harvesting (column B).

For chromosomal analysis, air-dried preparations were Giemsa-stained according to standard procedures. A minimum of 50 metaphase plates from coded slides for each culture were scored for chromosomal aberrations by two independent people. The chromosomal aberrations (CAs) included chromatid breaks, chromosome/isochromatid breaks, chromatid exchanges, dicentrics and ring chromosomes. Chromatid or isochromatid breaks were diagnosed when the distal chromatid segments were dislocated from the chromosomal axis or when the unstained segment was larger than the chromatid width.

Metaphases with more than $12 \mathrm{CAs}$ and/or fragmented chromosomes were excluded from the basic aberration scores. This exclusion was performed because of the difficulties in estimating the CAs score and because they probably do not remain in this proliferative compartment but rather activate the apoptosis program. Interchromatid bridges were also excluded from the aberration score. They correspond to unresolved chromatid bridges, which formed selectively in response to DOX, due to its effect as a DNA intercalating drug.

\section{Evaluation of $G 2$ timing}

G2 length was determined by the labelled metaphase technique (Mitchell et al., 1979), always in the absence of caffeine, so that ATM / ATR activity was undisturbed. The three control cultures (without genotoxic) and the 12 cultures corresponding to the four assayed drug concentrations were treated with a final $1 \mu \mathrm{Ci} / \mathrm{mL}[3 \mathrm{H}] \mathrm{TdR}$ concentration of $25 \mathrm{Ci} / \mathrm{mmol}$ specific activity (Amersham Radiochemical Center, UK) for 4, 5 and $6 \mathrm{~h}$ before harvesting. In the last $3 \mathrm{~h}$ of incubation, the 12 cultures treated with the genotoxic drugs at the different concentrations specified so that 3 cultures were obtained per each drug concentration. Metaphases were later accumulated by adding $0.2 \mu \mathrm{g} / \mathrm{mL}$ colcemid.

For autoradiography, air-dried preparations were dipped in EM-1 hypercoat emulsion RPN-41 (Amersham Biosciences, Uppsala, Sweden), placed in the dark at $4^{\circ} \mathrm{C}$, and after seven days of exposure, they were developed and then stained with $4 \%$ Giemsa. Scoring of at least 300 labelled metaphases was carried out in coded slides by two independent people. Mean G2 timing was estimated by taking into account the $[3 \mathrm{H}] \mathrm{TdR}$ incubation time at which $50 \%$ of the metaphases were [3H]labelled for each experimental condition. 
For each drug, the relationship between both chromosomal aberrations and G2 timing was assessed in function of the treatment concentration. The statistical significance of the mean values was estimated from the correlation coefficients (r) and the Student's t test. Minimum significance was considered to occur at a maximum $\mathrm{P}$ value of 0.05 . Only un-significant $\mathrm{P}$-values for correlation coefficients are denoted.

\section{RESULTS}

Endogenous (basal) DNA damage in the G2-prophase of control lymphocytes

The experimental protocol used in the present work restricts the exposure of human lymphocytes to antitumoral drugs to the last three hours before metaphase. As the mean length of the G2 phase in lymphocytes from control donors corresponded to $4.4 \pm 0.2$ hours, confirming previous results (Pincheira et al., 1993), the 3-hour treatment occupies an important part of $\mathrm{G} 2$, excluding only early $\mathrm{G} 2$.

In the absence of any genotoxic drug, lymphocytes displayed a few chromosomal aberrations both when caffeine was applied to cancel the checkpoint kinases ATM/ATR (6.3 \pm 1.0 CAs per 100 metaphases; column B in Table 1) and also when these kinases remained functional, i.e. in the absence of caffeine $(1.1 \pm 0.5$ CAs per 100 metaphases, column $\mathrm{A}$ in Table 1). The difference between the two values $(6.3$ minus 1.1 CAs/100 metaphases) corresponds to the DNA lesions repaired before their processing into CAs. Accordingly, when no functional ATM/ATR kinases were present, the reduction of 5.2 CAs per 100 metaphases (column C in Table 1) was due to the inhibition of DNA repair.

Efficiency of the genotoxic drugs on the induction of chromosomal aberrations

To select biological equivalent drug concentrations, we previously evaluated (data not shown) the concentration at which each drug treatment induced DNA damage (recorded as CAs) in slightly over $50 \%$ per 100 metaphases when the ATM/ATR kinases were cancelled by $5 \mathrm{mM}$ caffeine. In this way, both increases and decreases in DNA damage could be easily followed. Concentrations at which the presence of CAs per 100 metaphases was slightly over those corresponding to endogenous DNA damage $(6.3 \pm 1.0$ in first row, column B) were the lowest ones to be studied.

TABLE 1

DNA lesions in human lymphocytes under basal conditions and at different concentrations of DOX, ara C or HU, recorded as chromosomal aberrations (CAs) per 100 metaphases. (A) Unrepaired DNA lesions both in the presence of ATM/ATR checkpoint kinases and (B) after their caffeine-induced abrogation (Sarkaria et al. 1999). (C) DNA lesions repaired in the presence of the ATM/ATR kinases (no caffeine) (D) Estimated $\mathrm{G}_{2}$-prophase timings in hours and $(\mathbf{E})$ rates of DNA repair in CAs per 100 metaphases per hour.

\begin{tabular}{|c|c|c|c|c|c|c|}
\hline \multirow{2}{*}{\multicolumn{2}{|c|}{ Treatments }} & \multicolumn{2}{|c|}{ DNA lesions } & \multirow{2}{*}{$\begin{array}{c}\text { Repaired DNA lesions } \\
(+ \text { ATM/ATR }) \\
\text { C }=\mathbf{B}-\mathbf{A}\end{array}$} & \multirow{2}{*}{$\begin{array}{c}\mathrm{G}_{2} \text {-prophase } \\
\text { time (+ATM/ATR) } \\
\text { D }\end{array}$} & \multirow{2}{*}{$\begin{array}{c}\text { Rate of DNA } \\
\text { repair } \\
\mathrm{E}=\mathrm{C} / \mathrm{D}\end{array}$} \\
\hline & & $\begin{array}{c}(+\mathrm{ATM} / \mathrm{ATR}) \\
\mathbf{A}\end{array}$ & $\begin{array}{c}\text { (-ATM/ATR) } \\
\text { B }\end{array}$ & & & \\
\hline None $\left(^{*}\right)$ & & $1.1 \pm 0.5$ & $6.3 \pm 1$ & 5.2 & $4.4 \pm 0.1$ & 1.2 \\
\hline \multicolumn{7}{|c|}{$\operatorname{DOX}[\mu \mathrm{M}]$} \\
\hline 0.002 & & $3.8 \pm 1$ & $11.5 \pm 2$ & 7.7 & $4.5 \pm 0.1$ & 1.7 \\
\hline 0.02 & & $9.6 \pm 3$ & $17.3 \pm 3$ & 7.7 & $4.6 \pm 0.2$ & 1.7 \\
\hline 0.2 & & $19.2 \pm 3$ & $29.0 \pm 3$ & 9.6 & $5.1 \pm 0.2$ & 1.9 \\
\hline 2.0 & & $38.9 \pm 4$ & $57.0 \pm 6$ & 18.1 & $5.7 \pm 0.3$ & 3.2 \\
\hline \multicolumn{7}{|c|}{ ara $C[\mu \mathrm{M}]$} \\
\hline 0.01 & & $2.1 \pm 1$ & $7.7 \pm 2$ & 5.6 & $4.8 \pm 0.2$ & 1.2 \\
\hline 0.1 & & $6.8 \pm 2$ & $16.6 \pm 4$ & 9.8 & $5.1 \pm 0.2$ & 1.9 \\
\hline 1.0 & & $15.3 \pm 3$ & $28.7 \pm 5$ & 13.4 & $5.4 \pm 0.2$ & 2.5 \\
\hline 10.0 & & $37.9 \pm 6$ & $55.0 \pm 4$ & 17.1 & $5.9 \pm 0.3$ & 2.9 \\
\hline \multicolumn{7}{|c|}{$\mathbf{H U}[\mathrm{mM}]$} \\
\hline 1.0 & & $5.6 \pm 1$ & $12.2 \pm 2$ & 6.6 & $4.9 \pm 0.2$ & 1.3 \\
\hline 2.5 & & $13.4 \pm 3$ & $22.3 \pm 2$ & 8.9 & $5.1 \pm 0.2$ & 1.7 \\
\hline 5.0 & & $20.3 \pm 2$ & $35.6 \pm 5$ & 15.3 & $5.5 \pm 0.2$ & 2.8 \\
\hline 10.0 & & $31.9 \pm 6$ & $51.2 \pm 5$ & 19.3 & $5.7 \pm 0.3$ & 3.4 \\
\hline Highest $t$ & ggenous ratio in the column & - & - & 3.7 & 1.3 & 2.8 \\
\hline
\end{tabular}

The mean values \pm S.E. in the first row correspond to genotoxic-untreated lymphocytes from 30 healthy blood donors and represent the endogenous (basal) DNA damage produced mostly by oxidative metabolism. In contrast, the responses to each drug correspond to the mean values \pm SE estimated in 10 healthy individuals. Bold figures in $\mathbf{C}$, $\mathbf{D}$ and $\mathbf{E}$ represent the highest value recorded in each particular column. 
Obviously our choice did not ensure the strict equivalence of the concentrations of the different drug treatments, although the approach appeared as an acceptable estimate for their comparison.

First of all, the data showed that the range of concentrations at which the amount of CAs DOX and ara C induced changed from the minimum to the maximum selected concentration as much as 1,000 times, the corresponding maximum being $2.0 \mu \mathrm{M}$ and $10 \mu \mathrm{M}$ for DOX and ara $\mathrm{C}$, respectively. At the same concentrations, the minimum values for the induced CAs were $0.002 \mu \mathrm{M}$ for DOX and $0.01 \mu \mathrm{M}$ for ara C.

On the other hand, the range of active concentrations for HU was narrower than those for DOX or ara C. Thus, the selected minimum and maximum concentrations changed by a single order of magnitude ( $1 \mathrm{mM}$ and $10 \mathrm{mM}$, respectively).

Effect of the genotoxic drugs applied during G2-prophase in CAs frequency

The residual presence of CAs supports that the activation of the G2-prophase checkpoint was followed by checkpoint adaptation, a process that is also taking place under endogenous metabolic stress. Hence, the control cells when untreated with any genotoxic agent also overrode the checkpoint-induced G2-prophase block precociously, i.e. before the repair of DNA damage recorded as DSBs was over. In control cells, most of the DNA damage present was that induced by oxidative metabolism and, probably, by some stress induced by the in vitro cell culture. The CAs induced under these basal conditions remained constant while the growth conditions stayed unmodified.

When no caffeine was added, the frequency of CAs was reduced (column A in Table 1) in comparison to values obtained when the simultaneous ATM/ATR kinases activity was cancelled by caffeine (column B). However, the frequency of CAs increased in a drug concentration-dependent manner in both experimental conditions. The corresponding correlation coefficients $(r)$ for the regression lines recorded were 0.927 and 0.941 for DOX; 0.959 and 0.931 for ara C, and 0.985 and 0.988 for $\mathrm{HU}$, both in the presence and the absence of caffeine (columns A and B), respectively.

It was also estimated how much of the recorded chromosomal damage was produced in response to the antitumoral drugs and how much was produced by metabolism. Then the frequency of endogenous DNA damage recorded as CAs in the absence of caffeine (1.1 CAs/100 metaphases) was subtracted from the total frequency of CAs recorded at different drug concentrations (column A, Table 1). Equivalent estimations were carried out when caffeine was added (column B in Table 1).

The differences between frequency of CAs recorded in the presence (column B, Table 1) and in the absence of caffeine (column A) represent the amount of DNA lesions repaired through the activation of the G2-prophase checkpoint, i.e. in the presence of functional ATM/ATR kinases (no caffeine added). The estimated DNA lesions repaired are displayed in column $C$ of Table 1 . In the range of drug concentrations assayed, the amount of DNA being repaired increased linearly with drug concentration and then with the DNA damage induced by each specific treatment. The corresponding correlation coefficients for the recorded values were 0.966 , 0.825 and 0.975 for DOX, ara C and HU, respectively.

It was obvious that lymphocytes were able to repair nearly 4 times more DNA lesions in response to $10 \mathrm{mM}$ HU (last row, column $\mathrm{C}$ in Table 1) than when no genotoxic drug was applied (first row, in the same column). In this last situation, the only DNA damage present was that induced by oxidative metabolism and probably by some stress arising from the specific cell culture conditions. The CAs induced under these basal conditions remained constant while the growth conditions remained the same.

As recorded in column $\mathrm{C}$ of Table 1 , at the lowest drug concentrations, the level of CAs per 100 metaphases was similar in the ara C-treated cells to that in the control cells exposed only to the basal DNA damage. This basal value was increased in 1.4 under HU (6.6) and in 2.5 under DOX (7.7). Thus, at the lowest drug concentration most of the DNA lesions detected as CAs are formed in response to cell metabolism, while the remaining ones represent the processing products of the genotoxic effect that each drug treatment had during G2 -prophase.

Does the drug-induced DNA damage activate the G2-prophase checkpoint?

Three facts support the drug-induced activation of the studied checkpoint. First of all, though the DNA damage induced by either metabolism or by the applied drugs is repaired in the absence of the checkpoint ATM/ATR kinases, the activation of these kinases increases the amount of the DSB repaired.

Secondly, G2-prophase timings were recorded in proliferating lymphocytes from healthy individuals, both under control conditions and after the different treatments with the three anti-tumoral drugs. The data obtained (column D in Table 1) proved that the G2-prophase checkpoint pathway was activated by these drugs. Thus, the G2-prophase timings increased in a drug concentration-dependent manner in relation to the mean value recorded in the control lymphocytes untreated with any antitumoral drug. The correlation coefficients for the corresponding regression lines were 0.910, 0.808 and 0.909 for DOX, ara C and HU, respectively.

Lastly, the presence of CAs in metaphase after the treatment protocol here applied shows that checkpoint adaptation has taken place immediately after cell exposure to the genotoxic drugs during previous G2 and prophase. Only checkpoint adaptation is compatible with the entry into mitosis of the cells with still unrepaired DNA damage as CAs.

How much DNA damage induced in G2-prophase reaches metaphase still unrepaired?

Only a portion of the DNA damage produced in this part of the cycle by the oxidative metabolism or by the genotoxic drugs were repaired during G2-prophase. In fact, the unrepaired DNA constituted the most important portion of the total DNA lesions induced by the antitumoral treatments, as it occurred at the highest drug concentrations, where the amount of intranuclear DNA damage reached its maximum.

Thus, the amount of DNA damage increased from the residual 1.1 CAs per 100 metaphase recorded for the metabolic DNA damage produced in control conditions up to the maximum 38.9 CAs per 100 metaphases recorded in response 
to $2 \mu \mathrm{M}$ DOX treatment (column A in Table 1). The frequency of the DNA lesions remaining unrepaired because G2-prophase checkpoint adaptation increased with concentration in response to any of the three assayed genotoxic drugs (column A).

How is the efficiency of DNA repair modulated in lymphocytes?

The efficiency of DNA repair was estimated in the presence of each antitumoral drug treatment assayed during G2-prophase by taking into account the total DNA lesions repaired (column C, Table 1) and the mean G2 time that these cells need to repair them (column D in Table 1). The estimated values for the rate of DNA repair are shown in last column of Table 1 (column E). There is a linear increase in the rate of DNA repair in response to increased DNA damage, the correlation coefficients for the regression lines obtained were 0.956 for DOX and 0.969 for HU $(\mathrm{P}<0.05)$, while $\mathrm{r}$ was 0.761 for ara $\mathrm{C}(\mathrm{T}=2.1 ; 0.1>\mathrm{P}>0.05)$.

While the rate of repair for the endogenous damage produced by metabolism was of 1.2 CAs/100 metaphases per $\mathrm{h}$, it increased to $1.7 \mathrm{CAs} / 100$ metaphases per $\mathrm{h}$ for the two lowest DOX concentrations. It remained similar to the endogenous DNA damage for the minimum assayed concentrations of either ara C or HU.

On the other hand, the maximum levels reached by the rate of DNA repair took place at the highest assayed dose of each drug treatment. The maximum rates of DNA repair were 3.2, 2.9, and 3.4 CAs/100 metaphases per hour for DOX, ara $\mathrm{C}$ and HU, respectively (column E Table 1). Thus, the highest DNA repair rate was 2.8 fold higher than that displayed for the repair of the metabolism-induced DNA lesions. A modest 1.3 fold lengthening of the G2-prophase timing helps to increase DNA repair at the maximum drug concentrations assayed.

\section{DISCUSSION}

The presence of CAs in mitosis does not result from saturation of the cell repair capability in the previous G2-prophase. Hence, lymphocytes repaired up to 3.7 times more DNA lesions in response to $10 \mathrm{mM} \mathrm{HU}$ than under control conditions (where only endogenous lesions due to oxidative metabolism were present).

Adaptation to the G2-prophase checkpoint seems to be responsible for the appearance of CAs in mitosis. The recorded checkpoint-induced G2-prophase lengthening produced in response to the genotoxic drugs took place during those specific cycle phases. Furthermore, CAs increased the G2prophase checkpoint when abrogated by caffeine and when the cooperation of the checkpoint ATM/ATR kinases to repair the drug-induced DNA lesions was precluded (column B in Table 1 ). Finally, only adaptation to the G2-prophase checkpoint makes cells enter into mitosis before DNA repair is completed. The participation of ATM/ATR in the DNA repair and G2checkpoint mechanisms is well known since Sarkaria et al., (1999) reported it.

Checkpoint adaptation acquires a new significance when we think of it as a genetically programmed process for specific chromosomal situations. Thus, its evolutionary conservation favors experiments on microevolution in somatic cells where DNA has previously been damaged and this damage has activated the G2-prophase checkpoint. The trial and error assays on genome re-structuring that these experiments imply might improve the survival of the genetically modified progeny of each checkpoint-adapted cell, no matter how low the probability for increasing cell proliferation capability might be.

In cell cultures of ataxia telangiectasia patients, the spontaneous release from the G2 checkpoint-induced block (checkpoint adaptation) takes place when the presence of 3 or 4 chromosomal aberrations is ensured immediately following mitosis (Deckar et al., 2007). The threshold for spontaneous override of the G2-prophase checkpoint-induced cycle block increases with DNA damage, as shown here (by the present results). This is consistent with the fact that the threshold nearly trebled in the cells of patients with chromosome 21 trisomy that displays increased oxidative DNA damage (Pincheira et al., 2007).

In terms of energy cost, the G2-prophase checkpoint appears not to be as efficient as the previous one, which controls the $\mathrm{G} 1$ to $\mathrm{S}$ transition to transiently stop cell proliferation when the conditions of a cell are not adequate to deal safely with the new ones which the cell would face after irreversible onset of replication. However, the G2-prophase checkpoint has some features that increase its strategic value when compared to the G1 to S checkpoint. Thus, nuclear DNA damage reaches the G2 -prophase period after adaptation to previous intra $S$ checkpoints, so that the DNA lesions are far more abundant in late interphase than earlier in the cell cycle (Bernstein et al., 2002).

The G2-prophase checkpoint is even more crucial for cell survival in the already transformed cells than in the control ones. Thus, nearly half of all human tumors lack some of the active isoforms of the checkpoint protein p53, with the consequence that the previous checkpoint controlling the G1 to $S$ transition is constitutively inactive in them (Levine, 1997).

Genome instability: the unsuspected consequence of G2-prophase checkpoint operation

The response of normal lymphocytes to the three assayed antitumoral drugs allows us to conclude that activation of the G2-prophase checkpoint and the subsequent adaptation to the block that it induces in cell progression will be followed by the undue entry of cells with DNA breaks into metaphase.

As commented above, adaptation to the G2-prophase checkpoint but not to any other checkpoint challenges the cell in a unique way because there is a shift in the way that DSBs are repaired. This shift is produced both because of topoisomerase II-dependent premitotic chromatid individualization (Gimenez-Abian et al., 2000) and, also, because of chromosomal condensation, the main epigenetic process that represses gene expression in mitosis.

As a consequence, DSBs repair waits until G1 of the next cell cycle. Then, repair proceeds by an error-prone DNA recombination since these chromosomes are still unreplicated and the intact sequences in the homologous chromosomes are far apart and consequently not available to be copied for homologous recombination. Instead, the repair of DSBs in G1 has to proceed by non-homologous end-joining (NHEJ), as supported by the DNA-PKcs activity requirement for premature release of the G2 checkpoint (Lee et al., 1997).

The main drawback of the inaccurate joining of two unrelated free DNA ends located either in the same or different chromosomes is that it gives rise to chromosomal 
rearrangements such as translocations, dicentrics and other types of unstable chromatid rearrangements. Resolution of the ectopic DNA bonds produced by NHEJ repair of the druginduced DSBs takes place during the immediately following anaphase-telophase. This is mediated by random chromosomal cuts formed as a consequence of sister centromeres stretching towards opposite spindle poles. This results in the appearance of new DSBs that are again ectopically repaired in the following cell cycle. They are clonally inherited throughout subsequent cell cycles by following the universal DNA breakage-fusion-bridge cycle (McClintock, 1984) that ensures the clonal transmission of genomic instability.

In relation to the induction of genome instability in leukemia-treated children (Finette et al., 2000), it is worth remembering that microsatellite instability is brought about when the mismatch repair system in the G2-prophase is disturbed (Hawn et al., 1995).

\section{G2-prophase checkpoint adaptation and secondary neoplasias}

The results presented here show that the activation of the G2-prophase checkpoint favors, instead of preventing, the appearance of secondary neoplasias in the non-transformed cells of cancer patients, in a late response to previous antineoplastic drugs that damaged the DNA. Then, it cannot be assumed that checkpoints are tumor-suppressing mechanisms. Only when DNA damage overrides the cell's ability to repair itself does checkpoint-mediated induction of apoptosis take place and only then does the activation of the checkpoint result in a desirable antitumoral effect since every apoptotic cell is excluded from the next generation. At the drug concentrations and incubation times considered in this work, no apoptotic body programs responsible for apoptosis (Bernstein, 2002) were detected at late incubation times. Of these, only metaphases displaying many CAs probably endure a mitotic catastrophe and are excluded from the next cell generation.

The present results that a major objective for the development of safe antitumoral treatments based on genotoxic drugs is to rely on the reinforcement of the G2prophase checkpoint or, more precisely, on the prevention of adaptation to this specific checkpoint, which shifts DSB repair from error-free to error prone because of innate cell-cycle properties.

\section{CONFLICT OF INTEREST STATEMENT: NONE.}

\section{ACKNOWLEDGMENTS}

We thank Dr. Rodrigo Barra and Dr. Mauricio Bravo for their collaboration in obtaining blood samples from control individuals. We also thank Mrs. Beryl L. Walker for correcting the English manuscript. This work has been partially supported by Instituto de Ciencias Biomédicas, Facultad de Medicina, Universidad de Chile.

\section{REFERENCES}

BERNSTEIN C, BERNSTEIN H, PAYNE CM, GAREWAL H (2002) DNA repair/pro-apoptotic dual-role proteins in five major DNA repair pathways: fail-safe protection against carcinogenesis. A review. Mutat Res 511: 145-178.

DECKAR D, BIRRAUX J, KREMPLER A, THOUANDONG L, BEUCHER A, WALTER S, STAFF T, JEGGO P, LÖBRICH M (2007) Chromosome breakage after G2 checkpoint release. J Cell Biol 176: 749-755.

FINETTE BA, HOMANS AC, ALBERTINI RJ (2000) Emergence of genetic instability in children treated for leukemia. Science 288: 514-517.

GAJEWSKI E, GAUR S, AKMAN SA, MATSUMOTO L, VAN BALGOOY JN, DOROSHOW JH (2007) Oxidative DNA base damage in MCF-10A breast epithelial cells at clinically achievable concentrations of doxorubicin. Biochem Pharmacol 73: 1947-1956.

GIMÉNEZ-ABIÁN JF, CLARKE D.J, DEVLIN K, GIMÉNEZ-ABIÁN MI, DE LA TORRE C,JOHNSON RT, MULLINGER AM, DOWNES CS (2000) Premitotic chromosome individualization in mammalian cells depends on topoisomerase II activity. Chromosoma 109 235-244.

HAWN MT, UMAR A, CARETHERS JM, MARRA G, KUNKEL TA, BOLAND CR, KOI M (1995) Evidence for a connection between the mismatch repair system and the G2 cell cycle checkpoint. Cancer Res. 55: 37213725.

LEE SE, MITCHELL RA, CHENG A, HENDRICKSON EA (1997) Evidence for DNA PKdependent and -independent DNA double-strand break repair pathways in mammalian cells as a function of the cell cycle. Mol Cell Biol 17: 1425-1433.

LEVINE AJ (1997) p53, the cellular gatekeeper for growth and division. Cell 88: 323-331.

MCCLINTOCK B (1984) The significance of responses of the genome to challenge. Science 226: 729-801.

MIRZAYANS R, DIETRICH K, PATERSON MC (1993) Aphidicolin and 1- $\beta$ Darabinosylcytosine strongly inhibit transcriptionally active DNA repair in normal human fibroblasts. Carcinogenesis 14: 2621-2626.

MITCHELL JB, BEDFORD JS, BAILEY SM (1979) Dose-rate effect on the cell cycle survival of $\mathrm{S} 3$ HeLa cells. Radiat Res 79: 520-536.

MÜLLER A, BOITIER E, HU T, CARR GJ, LE FÈVRE AC, MARCHANDEAU JP, FLOR M, JEFFERSON F, AARDEMA MJ, THYBAUD V (2005) Laboratory variability does not preclude identification of biological functions impacted by hydroxyurea. Environ Mol Mutagen 46: 32-35.

PAULOVICH AG, TOCZYSKI DP, HARTWELL LH. (1997) When checkpoints fail. Cell 88: 315-321.

PINCHEIRA J, GALLO G, BRAVO M, LÓPEZ-SÁEZ JF (1993) G2 repair and ageing: Influence of donor age on chromosomal aberrations in human lymphocytes. Mutat Res 295: 55-62.

PINCHEIRA J, ROMERO P, MARCELAIN K, SALAZAR L, DE LA TORRE C (2007) G2 checkpoint-dependent DNA repair and its response to catalase in Down syndrome and control lymphocyte cultures. Cell Biol Int 31: 135-140.

SÁNCHEZ Y, WONG C, THOMA RS, RICHMAN R, WU Z, PIWNICAWORMS H, ELLEDGE SJ (1997) Conservation of the Chk1 pathway in mammals: linkage of DNA damage to Cdk regulation through Cdc25. Science 277:1497-1501.

SARKARIA JN, BUSBY EC, TIBBETTS RS, ROOS P, TAYA Y, KARNTIZ LM, ABRAHAM DRT (1999) Inhibition of ATM and ATR kinase activities by the radiosensitizing agent, caffeine. Cancer Res. 59: 4375-4382.

ZHANG WH, POH A, FANOUS AA, EASTMAN A (2008) DNA damageinduced $S$ phase arrest in human breast cancer depends on Chk1, but G2 arrest can occur independently of Chk1, Chk2 or MAPKAPK2. Cell Cycle 7: 1668-1677.

ZHOU BBS, ELLEDGE SJ (2000) The DNA damage responses: putting checkpoints in perspective. Nature 408 433-439. 